\title{
Ultrasound evaluation of complications after cardiovascular surgery in pediatric patients: A case series
}

\author{
Takahiro Hosokawa ${ }^{1}$, Sio Suzuki ${ }^{2}$, Yutaka Tanami ${ }^{1}$, Yumiko Sato ${ }^{1}$, Yoshihiro Ko ${ }^{3}$, \\ Koji Nomura $^{3}$, Eiji Oguma ${ }^{1}$, Yoshitake Yamada ${ }^{4}$
}

${ }^{1}$ Department of Radiology, Saitama Children's Medical Center, Saitama, ${ }^{2}$ Department of Cardiology, Saitama Children's Medical Center, Saitama, ${ }^{3}$ Department of Cardiovascular surgery, Saitama Children's Medical Center, Saitama, ${ }^{4}$ Department of Radiology, Keio University School of Medicine, Tokyo, Japan

\begin{abstract}
In contrast to computed tomography, ultrasound can be performed without radiation exposure, repeatedly performed by the patients' bedside. Hence, in this case series, we describe the evaluation of complications including hematoma, superficial surgical site infection, mediastinitis, and pseudoaneurysm associated mediastinitis using ultrasound in pediatric patients after cardiovascular surgery. To our knowledge, no previous reports have evaluated such complications using ultrasound. Ultrasound may be useful for the early diagnosis of these complications, and in the selection of subsequent examinations such as computed tomography, resulting in the early initiation of intervention.
\end{abstract}

Keywords; mediastinitis; cardiovascular surgery; congenital heart disease; ultrasound; surgical site infection

\section{Introduction}

In pediatric patients, cardiovascular surgery is mainly performed for congenital heart disease and two or three operations may be performed in the same case [1-3]. To date, some complications such as hematoma or surgical site infection including mediastinitis have been reported. These complications may be a life-threatening [4-8]. The

Received 16.04.2019 Accepted 11.06.2019

Med Ultrason

2020, Vol. 22, No 1, 108-113

Corresponding author: Takahiro Hosokawa, MD

Department of Radiology, Saitama Children's

Medical Center, 1-2 Shintoshin Chuo-ku

Saitama, Saitama, 330-8777 Japan

Phone: +81-48-601-2200

Fax: +81-48-601-2201

E-mail: snowglobe@infoseek.jp mediastinitis after cardiovascular surgery sometimes results in failure to complete all necessary procedures due to vein occlusion and strict adhesion and it may be result in more severe complications such as pseudoaneurysm [1,911]. Therefore, for pediatric patients, accurate diagnosis of these complications and early intervention are important.

Previous studies have reported that computed tomography $(\mathrm{CT})$ is useful for diagnosing complications such as such as hematoma, surgical site infection, mediastinitis and pseudoaneurysm associated mediastinitis [4,5,7,1216], However, to our knowledge, there have been no reports to evaluate these complications using ultrasound. In contrast to $\mathrm{CT}$, ultrasound can be performed without radiation exposure. In addition, because the patients do not have stable circulation after cardiovascular surgery, it may be difficult to move them from the intensive care unit; therefore ultrasound is an advantage as it can be performed by the patients' bedside [17,18]. 
Thus, the purpose of this case series demonstrated the utility of ultrasound imaging of various complications, including hematoma, surgical site infection, mediastinitis, and pseudoaneurysm associated mediastinitis in pediatric patients after cardiovascular surgery. In addition, we showed the sonogram with no complications in case 1 .

\section{Case series}

The Ethics committee of our institution approved this retrospective study, and the requirement to obtain informed consent from the patients was waived.

\section{Case 1}

Case 1 was a 5-month-old male patient with no eventful clinical course. He underwent cardiovascular surgery for an atrioventricular septal defect at the age of 4 months. After 1 month, ultrasound screening was performed for evaluation of mediastinitis. Mediastinitis was not detected on ultrasound (fig 1).

\section{Case 2}

A male patient aged 5 years and 4 months underwent cardiovascular surgeries at 3 months old and 3 years 8 months old for pulmonary artery atresia and a ventricular septal defect. He later experienced erythema and pain at the incision site at the age of 5 years and 4 months. Ultrasound revealed wire within the cystic region (fig 2). Hemorrhagic serous fluid was aspirated and hematoma around the wire was confirmed.

\section{Case 3}

Case 3 was a 1-month old male patient. Cardiac surgery for atrial/ventricular septal defect was performed at 1 month old. After 10 days, a precardiac mass was incidentally detected. Using ultrasound, a cystic lesion was revealed and $\mathrm{CT}$ was recommended. $\mathrm{CT}$ revealed a cystic mass with ring enhancement in the anterior wall (fig 3). To prevent invasion of the pulmonary artery, surgical treatment was selected. The patient was diagnosed with hematoma.

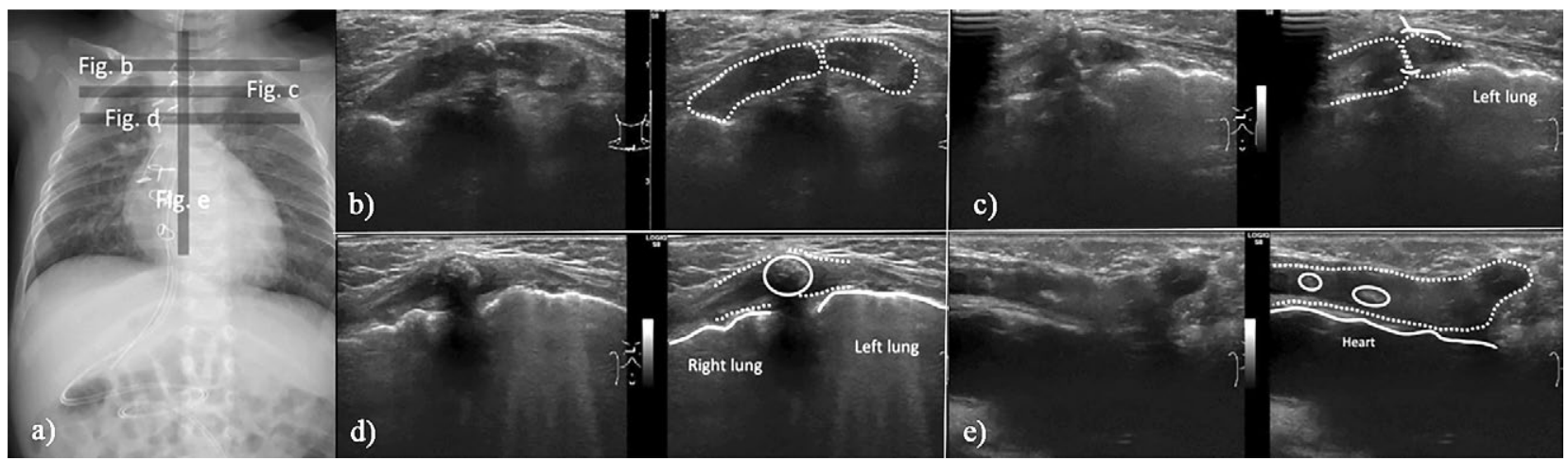

Fig 1. Imaging findings in a 5-month-old male patient with no eventful clinical course: a) frontal chest radiograph shows wires at the sternum; b) transverse sonogram at line b in image a shows that no dehiscence between the right and left separated sternum (dotted line). The sternum without ossification appears as a low echoic structure (dotted line); c) slightly lower site than that in b. Transverse sonogram at line $\mathrm{c}$ in a describing no dehiscence of the sternum (dotted line) and the wire appears as a hyperechoic line around the sternum (continuous line); d) slightly lower site than that in c, at line d, transverse sonogram showing ossification (continuous line) at the center of the sternum (dotted line); e) sagittal sonogram at the midline (line e in fig a) showing no fluid behind the sternum. There is no soft tissue mass between the sternum and the heart. The sternum is indicated by the dotted line. Ossified centers appear as high echoic structures (continuous line) within the sternum.

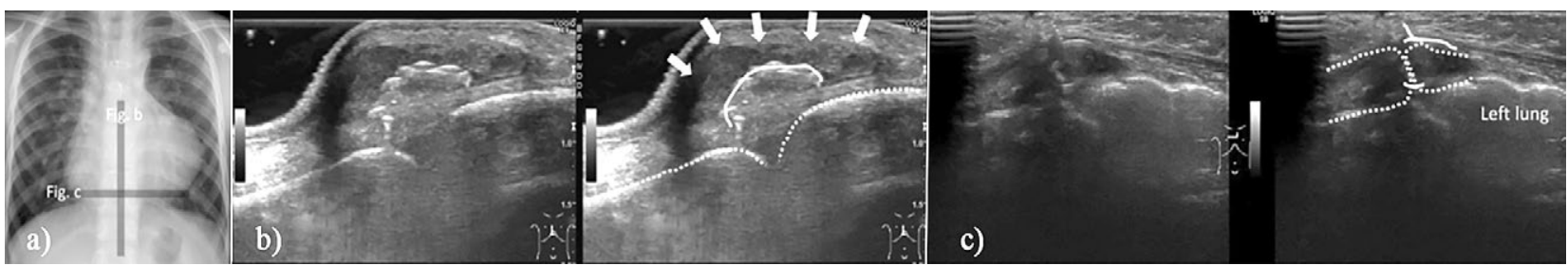

Fig 2. Imaging findings in case 2 : a) frontal chest radiograph shows wires at the sternum; b) sagittal sonogram at line $b$ in a describing a cystic mass (arrows) on the sternum (dotted line). The wire appears as a hyperechoic line (continuous line) within the cystic mass. The ossified sternum appears as a hyperechoic structure and acoustic shadows can be observed behind the sternum; c) transverse sonogram at line $\mathrm{c}$ in fig a showing a cystic mass (arrows) and the wire appears as a hyperechoic line (continuous line). The dotted line indicates the sternum. 


\section{Case 4}

Case 4 was a 1-month-old female patient. Surgical repair for transposition of the great arteries was performed at 10 days after birth. Twenty days after operation, consistent fever was observed and ultrasound evaluation was performed. Based on the ultrasound findings, surgical site infection was suspected and CT was recommended to determine the occurrence of deep sternal wound infection. CT revealed a low attenuation area behind and at the lower end of the sternum (fig 4). Although it was difficult to differentiate postoperative change from surgical site infection on CT, surgical aspiration was performed at the lower end of the sternum based on the sonographic findings. Surgical site infection with methicillin-resistant Staphylococcus aureus was confirmed.

\section{Case 5}

Case 5 was a female aged 4 years and 2 months. Surgical repair for Tetralogy of Fallot was performed at the age of 3 months. Subsequently, repeated surgical site infection was observed. She experienced erythema and pain at the incision site at 4 years and 2 months of age. Ultrasound revealed fluid collection at the incision site and CT was recommended. Based on the CT findings, incision drainage was selected and the patient was diagnosed with infection of the sternum (fig 5).

\section{Case 6}

In a male patient aged 1 year and 6 months after surgical repair for Ebstein's anomaly, superior vena cava stenosis was detected. Surgical repair was performed at

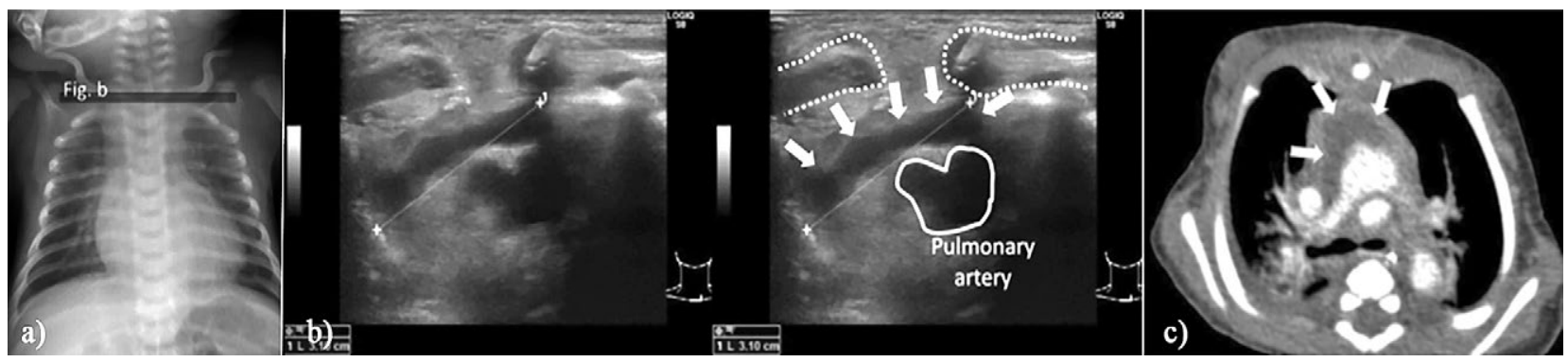

Fig 3. Imaging findings in case 3: a) frontal chest radiograph; b) transverse sonogram at line b in fig a showing fluid correction (arrows) anterior to the pulmonary artery (continuous line). The dotted line indicates the clavicle; c) axial enhanced computed tomography image describing fluid correction (arrows) with enhancement of the wall anterior to the pulmonary artery.
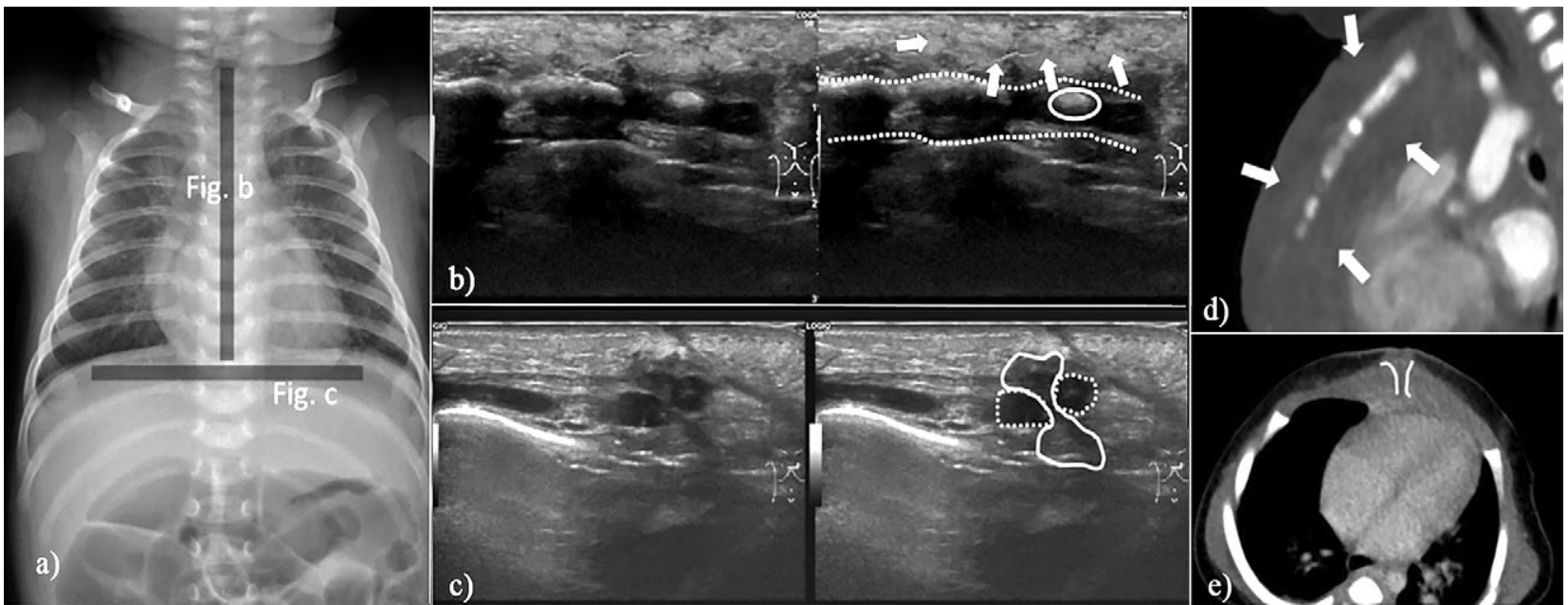

Fig 4. Imaging findings case 4: a) frontal chest radiograph showing wires at the sternum; b) sagittal sonogram at line b in fig a describing a hyperechoic area in the subcutaneous fat tissue (arrows). Fluid correction behind the sternum (dotted line) was not clearly revealed. The continuous line indicates the ossified center of the sternum. Right is a schematic presentation of image left; c) transverse sonogram at line $\mathrm{c}$ in fig a showing slight fluid correction (continuous line) between the right and left separated sternum (dotted line). Dehiscence can be observed between the right and left separated sternum (dotted line); d) sagittal contrast-enhanced computed tomography (CT) image describing no abscess formation behind the sternum. It was difficult to differentiate postoperative change from surgical site infection on the CT image; e) axial contrast-enhanced CT image at the lower end of the sternum shows low attenuation between the right and left separated sternum. 
1 year 6 months of age. From 10 days postoperatively, consistent fever was observed. Ultrasound was performed and fluid collection behind the sternum was revealed. CT was performed for evaluation. After diagnosis of mediastinitis, surgical debridement was performed. At 2 weeks postoperatively, a follow-up sonogram revealed a pseudoaneurysm within mediastinum. After performing ultrasound and CT, surgical repair was selected (fig 6).

\section{Discussions}

In this case series, we demonstrated the utility of ultrasound evaluation in various complications including hematoma, surgical site infection, mediastinitis and pseudoaneurysm associated mediastinitis in pediatric patients after cardiovascular surgery. Ultrasound was not only reported to be useful for accessing the abscess and for performing drainage, but may also be useful for the ear-
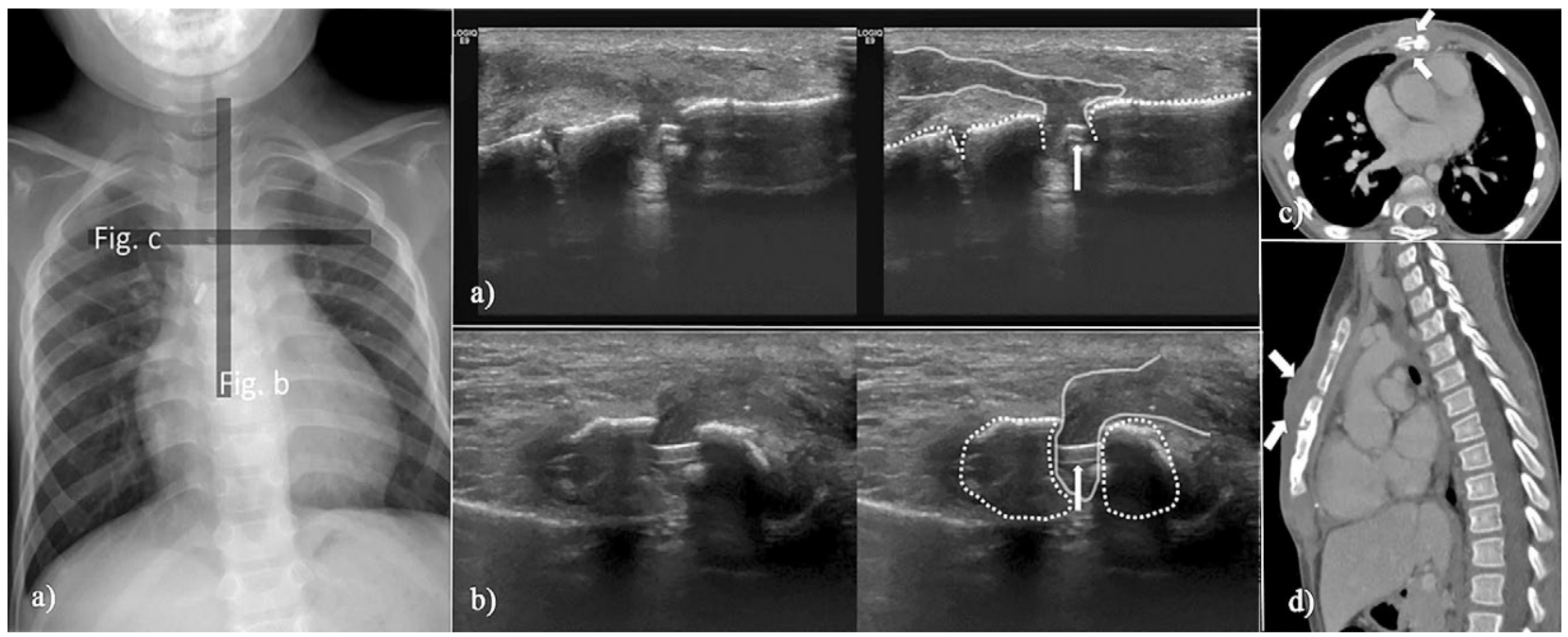

Fig 5. Imaging findings in case 5: a) chest radiograph showing a small wire at the sternum; b) sagittal sonogram at line b in a describing a wire (arrow) in the sternum (dotted line). Fluid correction is detected in the subcutaneous fat tissue (continuous line); c) transverse sonogram at line $\mathrm{c}$ in fig a showing dehiscence between the right and left separated sternum (dotted line). The wire appears as a hyperechoic line (arrow) and fluid correction is detected between the right and left separated sternum (continuous line); d) axial contrast-enhanced computed tomography (CT) image describing low attenuation around the wire and sternum dehiscence; e) sagittal contrast-enhanced CT image showing low attenuation in the subcutaneous fat tissue.

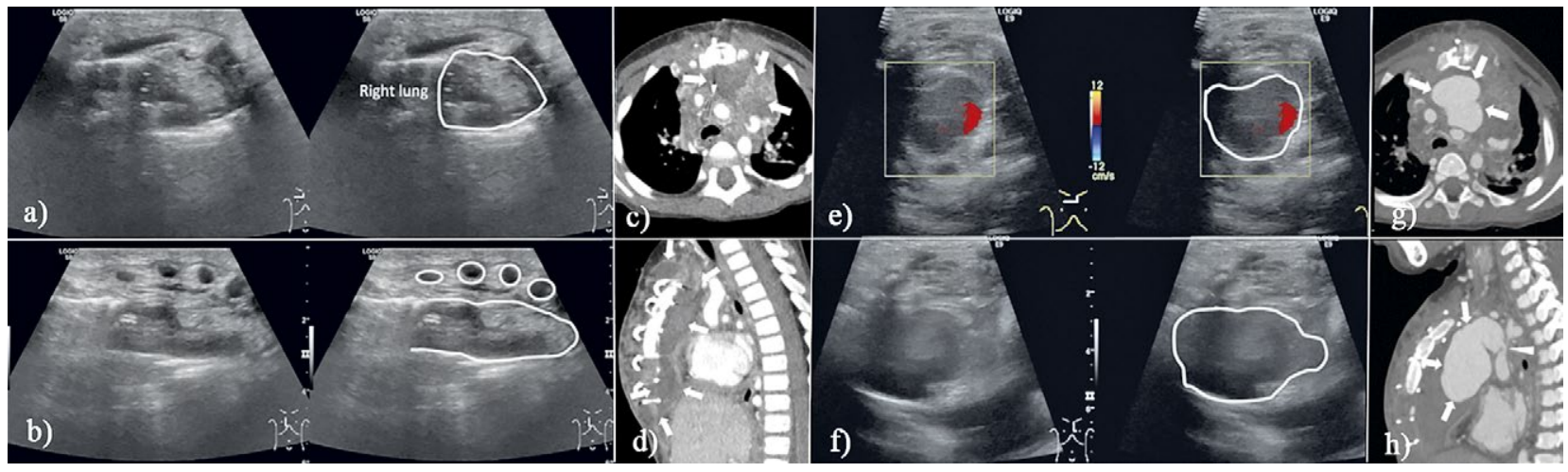

Fig 6. Imaging findings in case 6 who underwent surgical treatment for superior vena cava stenosis before 10 days (a-d) and before 2 weeks (e-h): a) transverse sonogram showing fluid correction with debris (continuous line); b) sagittal sonogram describing fluid correction between the anterior wall of the thorax and the heart. The circles indicate the ribs; c) axial contrast-enhanced computed tomography (CT) image showing destruction of the sternum and abscess formation (arrows); d) sagittal contrast-enhanced CT image showing fluid correction behind the sternum (arrows); e) transverse sonogram describing a pseudoaneurysm (surrounded continuous line) with internal turbulent flow (red color); f) sagittal sonogram revealing a pseudoaneurysm (continuous line); g) axial contrastenhanced CT image showing a pseudoaneurysm (arrows) connected to the ascending aorta; $h$ ) sagittal contrast-enhanced CT image describing a pseudoaneurysm (arrows) connected to the ascending aorta with a narrow neck (arrowhead). 
ly diagnosis of complications in pediatric patients who have undergone cardiac surgery $[19,20]$. In addition, ultrasound can be performed repeatedly and this advantage was important for the detection of complications such as pseudoaneurysm, which were sometimes not accompanied by associated symptoms (see case 6). According to our current cases, ultrasound may be recommended in pediatric patients in whom various complications are suspected after cardiovascular surgery.

In our pediatric patients, the fluid collection behind insufficient sternal ossification could be visualized by ultrasound (cases 3, 4, and 6), whereas ossified bone structures in adult patients makes acoustic shadows and blind areas. In previous studies, sternal destruction and fluid correction were reported to be important factors in the diagnosis of mediastinitis [21-23]. Although bone destruction may be difficult to detect with ultrasound, fluid collection or dehiscence can be detected with this modality between the right and left separated sternum (case 4, 5).

However, despite surgeon-led evaluation of the risk factors and improved surgical timing or devices to prevent complications, it can still occur [24-29]. In the short interval between the operation and the imaging examination, the differentiation of findings of mediastinitis and operative change is reported to be difficult $[4,5,12]$. In current cases, there were two cases of hematoma rather than mediastinitis (case 2,3). The differentiation of infection and hematoma may also be difficult by using imaging modalities such as ultrasound and $\mathrm{CT}$ alone. Physical examination findings of fever and erythema at the incision site are important for the diagnosis of mediastinitis (case 4, 5) [8,30].

Although the current case series demonstrates the utility of ultrasound for the evaluation of complications after cardiovascular surgery in pediatric patients, there have been no systematic studies of the use of ultrasound in the diagnosis of mediastinitis in pediatric patients. Further studies including ultrasound and other modalities, such as magnetic resonance imaging, may be required to support our results.

\section{Conclusion}

In this case series, we demonstrated the utility of ultrasound evaluation of various complications, including hematoma, surgical site infection, mediastinitis, and pseudoaneurysm associated mediastinitis in pediatric patients after cardiovascular surgery. According to our current cases, ultrasound may be recommended in pediatric patients in whom certain complications after cardiovascular surgery are suspected.

Conflict of interest: none

\section{References}

1. Laussen PC, Neonates with congenital heart disease. Curr Opin Pediatr 2001;13:220-226.

2. Reddy VM. Low birth weight and very low birth weight neonates with congenital heart disease: timing of surgery, reasons for delaying or not delaying surgery. Semin Thorac Cardiovasc Surg Pediatr Card Surg Annu 2013;16:13-20.

3. Wernovsky G, Ghanayem N, Ohye RG,et al. Hypoplastic left heart syndrome: consensus and controversies in 2007. Cardiol Young 2007; 17 Suppl 2:75-86.

4. Jolles H, Henry DA, Roberson JP, Cole TJ, Spratt JA. Mediastinitis following median sternotomy: CT findings. Radiology 1996;201:463-466.

5. Yamashiro T, Kamiya H, Murayama S, et al. Infectious mediastinitis after cardiovascular surgery: role of computed tomography. Radiat Med 2008;26:343-347.

6. Carrol CL, Jeffrey RB Jr, Federle MP, Vernacchia FS. CT evaluation of mediastinal infections. J Comput Assist Tomogr 1987;11:449-454.

7. Yamaguchi H, Yamauchi H, Yamada T, Ariyoshi T, Aikawa $\mathrm{H}$, Kato Y. Diagnostic validity of computed tomography for mediastinitis after cardiac surgery. Ann Thorac Cardiovasc Surg 2001; 7:94-98.

8. Tortoriello TA, Friedman JD, McKenzie ED, et al. Mediastinitis after pediatric cardiac surgery: a 15-year experience at a single institution. Ann Thorac Surg 2003;76:1655-1660.

9. Pena E, Fontan L, Blanco M. [Superior vena cava syndrome and collateral circulation in the abdominal wall by a fibrosing mediastinitis. Med Clin (Barc) 2012;139:e9.

10. Chevalier AT, Khaja MS, Yang B. Surgical Management of Necrotizing Mediastinitis With Large Aortic Pseudoaneurysm. Ann Thorac Surg 2016;101:e143-e145.

11. Mezzetto L, Treppiedi E, Scorsone L, et al. Thoracic Aortic Pseudoaneurysm after Esophageal Perforation and Mediastinitis Caused by Accidental Ingestion of a Mutton Bone: A Case Report on Staged Endoscopic and Endovascular Treatments. Ann Vasc Surg 2016;30:307.e15-e19.

12. Goodman LR, Kay HR, Teplick SK, Mundth ED. Complications of median sternotomy: computed tomographic evaluation. AJR Am J Roentgenol 1983;141:225-230.

13. Hélénon $\mathrm{O}$, Folinais $\mathrm{D}$, Cornud $\mathrm{F}$, et al. X-ray computed tomography in the course of mediastinitis after sternostomy for heart surgery. J Radiol 1987;68:237-245.

14. Kay HR, Goodman LR, Teplick SK, Mundth ED. Use of computed tomography to assess mediastinal complications after median sternotomy. Ann Thorac Surg 1983;36:706714.

15. Maddern IR, Goodman LR, Almassi GH, Haasler GB, McManus RP, Olinger GN. CT after reconstructive repair of the sternum and chest wall. Radiology 1993;186:665-670.

16. Risnes I, Abdelnoor M, Ulimoen G, et al. Mediastinitis after coronary artery bypass grafting increases the incidence of left internal mammary artery obstruction. Int Wound J 2014;11:594-600

17. Hosokawa T, Yamada Y, Tanami Y, Sato Y, Hosokawa M, Oguma E. Predictors of prognosis in children with 
portal venous gas detected by ultrasound. Med Ultrason, 2019;21:30-36.

18. Hosokawa T, Yamada Y, Takahashi H, et al. Postnatal U1trasound to Determine the Surgical Strategy for Congenital Diaphragmatic Hernia. J Ultrasound Med 2019 Jan 16. doi:10.1002/jum.14929.

19. Conzo G, Docimo G, Palazzo A, et al. The role of percutaneous US-guided drainage in the treatment of splenic abscess. Case report and review of the literature. Ann Ital Chir 2012;83:433-436.

20. Inoue M, Nakatsuka S, Yashiro H, et al., Lymphatic Intervention for Various Types of Lymphorrhea: Access and Treatment. Radiographics 2016;36:2199-2211.

21. Gaspari R, Dayno M, Briones J, Blehar D. Comparison of computerized tomography and ultrasound for diagnosing soft tissue abscesses. Crit Ultrasound J 2012;4:5.

22. Gan YU, Lam SL. Imaging findings in acute neck infection due to pyriform sinus fistula. Ann Acad Med Singapore 2004;33:636-640.

23. Wang CL, Guo XJ, Qiu SB, et al. Diagnosis of bacterial hepatic abscess by CT. Hepatobiliary Pancreat Dis Int 2007;6:271-275.

24. Sahin M. The role of topical Genta Fleece HD and gentamicin spray in prevention of sternum wound infections after open heart surgery: a comparative study. Arch Med Sci Atheroscler Dis 2018;3:e29-e34.

25. Nelson-McMillan K, Hornik CP, He X, et al. Delayed Sternal Closure in Infant Heart Surgery-The Importance of Where and When: An Analysis of the STS Congenital Heart Surgery Database. Ann Thorac Surg 2016;102:1565-1572.

26. Madej T, Plötze K, Birkner C, Jatzwauk L, Klaus M, Waldow T. Reducing Mediastinitis after Sternotomy with Combined Chlorhexidine-Isopropyl Alcohol Skin Disinfection: Analysis of 3,000 Patients. Surg Infect (Larchmt) 2016;17:552-556.

27. Katayanagi T. Nasal methicillin-resistant S. aureus is a major risk for mediastinitis in pediatric cardiac surgery. Ann Thorac Cardiovasc Surg 2015;21:37-44.

28. Abu-Omar Y, Kocher GJ, Bosco P, et al. European Association for Cardio-Thoracic Surgery expert consensus statement on the prevention and management of mediastinitis. Eur J Cardiothorac Surg 2017;51:10-29.

29. Anslot C, Hulin S, Durandy Y. Postoperative mediastinitis in children: improvement of simple primary closed drainage. Ann Thorac Surg 2007;84:423-428.

30. Durandy Y. Mediastinitis in pediatric cardiac surgery: Prevention, diagnosis and treatment. World J Cardiol 2010;2:391-398. 